\title{
The mass hierarchy in the relativistic uniform system
}

\author{
Sergey G. Fedosin \\ PO box 614088, Sviazeva str. 22-79, Perm, Perm Krai, Russia \\ E-mail: fedosin@hotmail.com
}

The analysis of the relativistic uniform system in the framework of the covariant theory of gravitation leads to five different masses of the physical system, in which the particles are bound together by means of the electromagnetic and gravitational fields, the acceleration field and the pressure field. In this case it turns out that the system's gravitational mass is equal to the sum of the invariant masses of all the system's particles, and the system's inertial mass is less than the gravitational mass by the value of the system's binding mass-energy.

Keywords: relativistic uniform system; gravitational mass; inertial mass; invariant mass; energy of system.

\section{Introduction}

For each physical system, we can introduce several types of masses, each of which characterizes the system in its own way. The inertial or invariant mass, which is related to the system's relativistic energy by a multiplier in the form of the Lorentz factor and the speed of light squared, is widely known. If the selected reference frame is stationary relative to the center of momentum of the physical system under consideration, the Lorentz factor of the system becomes equal to unity. In such a reference frame, there still remains proper motion of the particles and fields, and it is necessary to take into account the Lorentz factors of individual particles. When calculating the relativistic energy, it turns out that contribution to the inertial mass is made both by the energies of the particles in the fields acting on them, and by the energies of the fields themselves. In particular, the energy of the gravitational and electromagnetic fields must be calculated both in the empty space between the particles and even outside the system. Moreover, the matter particles, including small particles with the size of an individual atom, contain almost only empty space. This is due to the fact that almost the entire mass of an atom is contained in a small atomic nucleus. Therefore, the field energy must be calculated also inside the matter particles. In connection with this, when 
calculating the field energy, as a rule, integration is performed over the entire volume of the space of the reference frame under consideration.

In classical mechanics, the inertial mass is not only associated with the relativistic energy, but also determines the acceleration of bodies under the action of the forces applied to the system. A certain exception is the gravitation force. We can assume that the gravitational force acting from body 1 on body 2 is proportional to the active gravitational mass of body 1 and the passive gravitational mass of body 2. These masses are interpreted differently depending on the theory of gravitation used. For example, in the general theory of relativity, the active and passive gravitational masses do not differ from each other, and the inertial mass is equal to the gravitational mass of the system.

There is at least one more mass, often used in astrophysics. This is the total mass of baryons that make up the system, to which the total mass of the available electrons is added for accuracy. Indeed, the matter of planets and stars consists of nucleons and electrons, which can get connected with each other, forming separate atomic nuclei, atoms and ions, as well as the matter in different phase states. Obviously, the total mass of baryons and electrons is not equal to the inertial mass of the system, since during formation of a bound system, it releases the binding energy, and the relativistic energy of the system decreases.

Next we will consider the question of how the above-mentioned masses are interrelated in the covariant theory of gravitation. In doing so, we will use a physical model of particles and fields, which represents the relativistic uniform system. For the sake of simplicity, only the main four fields will be taken into account, including the electromagnetic and gravitational fields, the acceleration field and the pressure field. Each of these fields is assumed to be a vector field of Maxwell-type. All these fields are considered as the corresponding components of the general field [1], [2], and are used to describe macroscopic systems [3].

This approach was used earlier to estimate various masses of the system of particles and fields of a spherical shape in [4], [5]. In the system under consideration the particles move chaotically and so closely to each other that the approximation of continuous matter distribution can be applied. In this case, some part of the particles is charged, and the charge density distribution is similar to the mass density distribution.

Using now the properties of the relativistic uniform system [6], we will calculate the more accurate values of the system's masses, and will compare them by arranging in a certain sequence. The starting point of our reasoning will be the formula for the relativistic energy of a physical system with continuous matter distribution [7]: 


$$
\begin{aligned}
& E=\frac{1}{c} \int\left(\rho_{0} \psi+\rho_{0 q} \varphi+\rho_{0} \vartheta+\rho_{0} \wp\right) u^{0} \sqrt{-g} d x^{1} d x^{2} d x^{3}- \\
& -\int\left(\frac{c^{2}}{16 \pi G} \Phi_{\mu \nu} \Phi^{\mu \nu}-\frac{1}{4 \mu_{0}} F_{\mu \nu} F^{\mu \nu}-\frac{c^{2}}{16 \pi \eta} u_{\mu \nu} u^{\mu \nu}-\frac{c^{2}}{16 \pi \sigma} f_{\mu \nu} f^{\mu \nu}\right) \sqrt{-g} d x^{1} d x^{2} d x^{3} .
\end{aligned}
$$

In (1) $c$ is the speed of light; $\rho_{0}$ and $\rho_{0 q}$ denote invariant mass and charge densities, respectively; $\psi, \varphi, \vartheta$ and $\wp$ are the scalar potentials of gravitational and electromagnetic fields, acceleration field and pressure field, respectively; $\Phi_{\mu \nu}, F_{\mu \nu}, u_{\mu \nu}$ and $f_{\mu \nu}$ are the tensors of these fields, respectively; $u^{0}$ is the time component of the four-velocity of the element of matter; $g$ is the determinant of the metric tensor; $d x^{1} d x^{2} d x^{3}$ is the product of differentials of the spatial coordinates; $G$ is the gravitational constant; $\mu_{0}$ is the magnetic constant; $\eta$ is the acceleration field coefficient; $\sigma$ is the pressure field coefficient.

Formula (1) is valid in the approximation, in which the potentials and field tensors, found as superpositions of the contributions of the entire set of particles, do not depend on the velocities of individual particles of the system. Using this formula in [5], the energy for the equilibrium system of a spherical shape with the continuously distributed chaotically moving matter, taking into account the fields' energy, was calculated explicitly with an accuracy up to the terms that do not contain the speed of light squared in the denominators. Taking into account the corrections made in [8], the energy is equal to [9]:

$$
\begin{aligned}
& E=M c^{2} \approx m c^{2} \gamma_{c}^{2}-\frac{3 \eta m^{2} \gamma_{c}^{2}}{5 a}-\frac{6 G m^{2} \gamma_{c}^{2}}{5 a}+\frac{3 q^{2} \gamma_{c}^{2}}{10 \pi \varepsilon_{0} a}+m \wp_{c} \gamma_{c}-\frac{3 \sigma m^{2} \gamma_{c}^{2}}{10 a}+ \\
& +\frac{3 G m^{2} \gamma_{c}^{2}}{5 a}-\frac{3 q^{2} \gamma_{c}^{2}}{20 \pi \varepsilon_{0} a}-\frac{\eta m^{2} \gamma_{c}^{2}}{10 a}-\frac{\sigma m^{2} \gamma_{c}^{2}}{10 a} .
\end{aligned}
$$

The last four terms in (2) define the field energies, the quantity $M$ is the invariant inertial mass of the system, the mass $m$ is the product of the mass density $\rho_{0}$ by the volume of the sphere with the particles, $\gamma_{c}$ is the Lorentz factor of the particles in the center of the sphere, $a$ denotes the radius of the sphere, the charge $q$ is defined as the product of the charge density $\rho_{0 q}$ by the volume of the sphere, $\varepsilon_{0}$ is the electric constant, $\wp_{c}$ is the scalar potential of pressure field in the center of sphere. 


\section{Normalization of the mass and energy}

The system's energy is an integral quantity, it contains contributions from the energy of particles and fields, and in the general case requires normalization. It is convenient to normalize the energy with the help of the cosmological constant $\Lambda$, which is part of the system's Lagrangian. Formula (1) for the four main fields was obtained under the following condition [7]:

$$
-c k \Lambda=D_{\mu} J^{\mu}+A_{\mu} j^{\mu}+U_{\mu} J^{\mu}+\pi_{\mu} J^{\mu},
$$

where $D_{\mu}=\left(\frac{\psi}{c},-\mathbf{D}\right)$ is the four-potential of gravitational field in the framework of the covariant theory of gravitation,

$J^{\mu}=\rho_{0} u^{\mu}$ is the mass four-current,

$\rho_{0}$ is the invariant mass density,

$u^{\mu}$ is the four-velocity of point particle,

$A_{\mu}=\left(\frac{\varphi}{c},-\mathbf{A}\right)$ is the electromagnetic four-potential,

$j^{\mu}=\rho_{0 q} u^{\mu}$ is the charge four-current,

$\rho_{0 q}$ is the invariant charge density,

$U_{\mu}=\left(\frac{\vartheta}{c},-\mathbf{U}\right)$ is the four-potential of acceleration field,

$\pi_{\mu}=\left(\frac{\wp}{c},-\boldsymbol{\Pi}\right)$ is the four-potential of pressure field,

$\mathbf{D}, \mathbf{A}, \mathbf{U}$ and $\boldsymbol{\Pi}$ are the vector potentials of gravitational and electromagnetic fields, acceleration field and pressure field, respectively.

With the help of (3) we simplified the equations for the metric and performed gauge of the relativistic energy so that $\Lambda$ and the scalar curvature $R$ disappear from both of them.

Within the framework of the special theory of relativity the four-currents are: $j^{\mu}=\rho_{0 q} u^{\mu}=\rho_{0 q}(\gamma c, \gamma \mathbf{v}), J^{\mu}=\rho_{0} u^{\mu}=\rho_{0}(\gamma c, \gamma \mathbf{v})$, where $\gamma$ is the Lorentz factor, $\mathbf{v}$ is the velocity of system's particles. We substitute this in (3): 
$-c k \Lambda=\gamma \rho_{0 q} \varphi-\gamma \rho_{0 q} \mathbf{A} \cdot \mathbf{v}+\gamma \rho_{0} \psi-\gamma \rho_{0} \mathbf{D} \cdot \mathbf{v}+\gamma \rho_{0} \vartheta-\gamma \rho_{0} \mathbf{U} \cdot \mathbf{v}+\gamma \rho_{0} \wp-\gamma \rho_{0} \boldsymbol{\Pi} \cdot \mathbf{v}$.

Within the limit of low velocities we can neglect the terms, containing the particle velocity $\mathbf{v}$ and the vector potentials. Then in (4) the Lorentz factor is $\gamma \approx 1$ and only the terms with the scalar potentials of the fields are left. For the particles scattered to infinity in cosmic space we can assume that these potentials arise only from the proper fields of the particles and are the potentials averaged with respect to the volume of the particles. In this case $\vartheta \approx c^{2}$ and we can write:

$$
-c k \Lambda \approx \rho_{0 q} \varphi+\rho_{0} \psi+\rho_{0} c^{2}+\rho_{0} \wp .
$$

The scalar potentials of the fields inside the sphere were found in [10]. After simplification they look as follows:

$$
\begin{aligned}
& \varphi \approx \frac{\gamma_{c} \rho_{0 q}\left(3 a^{2}-r^{2}\right)}{6 \varepsilon_{0}}, \quad \psi \approx-\frac{2 \pi G \gamma_{c} \rho_{0}\left(3 a^{2}-r^{2}\right)}{3}, \\
& \vartheta \approx c^{2} \gamma_{c}-\frac{2 \pi \eta \rho_{0} r^{2} \gamma_{c}}{3}, \quad \wp \approx \wp_{c}-\frac{2 \pi \sigma \rho_{0} r^{2} \gamma_{c}}{3} .
\end{aligned}
$$

This shows that the cosmological constant is defined by the rest energy density of the particles with addition of the energy density of the particles in their proper fields. If we average (5) with respect to the cosmic space, then at $-c k=\frac{c^{4}}{16 \pi G \beta}$, where $\beta$ is a constant of the order of unity, the value $\Lambda \approx \frac{16 \pi G \rho_{0} \beta}{c^{2}}$ is obtained. Substituting here the standard estimate of the cosmological constant $\Lambda \approx 10^{-52} \mathrm{~m}^{-2}$, we find the corresponding matter density: $\rho_{0} \approx 3 \times 10^{-27} \mathrm{~kg} / \mathrm{m}$, which is sufficiently close to the observed average mass density.

Suppose now that a certain amount of matter under the action of gravitation is drawn together into such objects as gas clouds, and then into planets and stars. In [5] we integrated (4) over the volume of a fixed sphere, filled with the particles, which were held in that state by the action of gravitation and the electromagnetic field, taking into account the internal 
acceleration field and pressure field. If we denote by $m^{\prime}$ the gauge mass-energy of the system's particles, associated with the cosmological constant in (4), the integration results, in view of more precise values of the energies of particles in the potentials of the gravitational and electromagnetic fields [8,9], would be as follows:

$$
m^{\prime} c^{2} \approx m c^{2} \gamma_{c}^{2}-\frac{3 \eta m^{2} \gamma_{c}^{2}}{5 a}-\frac{6 G m^{2} \gamma_{c}^{2}}{5 a}+\frac{3 q^{2} \gamma_{c}^{2}}{10 \pi \varepsilon_{0} a}+m \wp_{c} \gamma_{c}-\frac{3 \sigma m^{2} \gamma_{c}^{2}}{10 a}
$$

The terms on the right-hand side of (6) are contained in (2), so that the energy can be written as follows:

$$
E \approx m^{\prime} c^{2}+\frac{3 G m^{2} \gamma_{c}^{2}}{5 a}-\frac{3 q^{2} \gamma_{c}^{2}}{20 \pi \varepsilon_{0} a}-\frac{\eta m^{2} \gamma_{c}^{2}}{10 a}-\frac{\sigma m^{2} \gamma_{c}^{2}}{10 a}
$$

In (2), the mass $m$ and the charge $q$ are used as auxiliary quantities. However, outside the sphere, the potentials of the gravitational and electromagnetic fields are determined with the help of the mass $m_{b}$ and the electric charge $q_{b}$. For these masses and charges we obtain [5], [11]:

$$
m \gamma_{c} \approx m_{b}+\frac{3 \eta m_{b}^{2}}{10 a c^{2} \gamma_{c}}, \quad q \gamma_{c} \approx q_{b}+\frac{3 \eta m_{b} q_{b}}{10 a c^{2} \gamma_{c}}
$$

The equation of motion of the matter inside the sphere [3] and the generalized Poynting theorem [9] lead to the following relation between the fields' coefficients:

$$
\eta+\sigma=G-\frac{\rho_{0 q}^{2}}{4 \pi \varepsilon_{0} \rho_{0}^{2}}
$$

In view of (8) and (9), the expression (7) can be rewritten as follows:

$$
E=M c^{2} \approx m^{\prime} c^{2}+\frac{G m^{2} \gamma_{c}^{2}}{2 a}-\frac{q^{2} \gamma_{c}^{2}}{8 \pi \varepsilon_{0} a} \approx m^{\prime} c^{2}+\frac{G m_{b}^{2}}{2 a}-\frac{q_{b}^{2}}{8 \pi \varepsilon_{0} a} .
$$


This shows that the relativistic energy of the system is equal to the total energy of the system's particles in proper fields, from which we should subtract the energy of the gravitational and electromagnetic fields outside the volume, filled with the system's particles.

\section{Comparison of the masses}

In [12], we calculated the total rest energy $W_{b}$ of the sphere's particles from the viewpoint of the observer associated with the sphere, the kinetic energy $E_{k}$, the scalar potential $\wp_{c}$ of the pressure field at the center of the sphere, and found the relations between the fields' coefficients, as well as the Lorentz factor $\gamma_{c}$ for the particles' motion at the center of the sphere :

$$
\begin{gathered}
W_{b}=m_{b} c^{2} \approx m c^{2} \gamma_{c}-\frac{3 \eta m^{2} \gamma_{c}}{10 a}, \quad E_{k} \approx m c^{2} \gamma_{c}^{2}-\frac{3 \eta m^{2} \gamma_{c}^{2}}{5 a}-W_{b} \approx \frac{27 \eta m^{2} \gamma_{c}}{20 \sqrt{14} a}, \\
\wp_{c} \approx \frac{\eta m}{5 a}\left(1+\frac{9}{2 \sqrt{14}}\right), \quad \eta=\frac{3}{5}\left(G-\frac{\rho_{0 q}^{2}}{4 \pi \varepsilon_{0} \rho_{0}^{2}}\right), \quad \sigma=\frac{2}{5}\left(G-\frac{\rho_{0 q}^{2}}{4 \pi \varepsilon_{0} \rho_{0}^{2}}\right), \\
\sigma=\frac{2 \eta}{3}, \quad \frac{G m^{2} \gamma_{c}^{2}}{2 a}-\frac{q^{2} \gamma_{c}^{2}}{8 \pi \varepsilon_{0} a} \approx \frac{5 \eta m^{2} \gamma_{c}^{2}}{6 a}, \quad \gamma_{c}=\frac{1}{\sqrt{1-v_{c}^{2} / c^{2}}} \approx 1+\frac{3 \eta m}{10 a c^{2}}\left(1+\frac{9}{2 \sqrt{14}}\right), \\
E=M c^{2} \approx m_{b} c^{2}-\frac{1}{10 \gamma_{c}}\left(7-\frac{27}{2 \sqrt{14}}\right)\left(\frac{G m_{b}^{2}}{a}-\frac{q_{b}^{2}}{4 \pi \varepsilon_{0} a}\right) .
\end{gathered}
$$

In (11), the energy $E$ is expressed in terms of mass $m_{b}$ and charge $q_{b}$. From comparison of (8), (10) and (11) we obtain relations for the mass-energies of the system under consideration:

$$
M=m^{\prime}+\frac{G m_{b}^{2}}{2 a c^{2}}-\frac{q_{b}^{2}}{8 \pi \varepsilon_{0} a c^{2}}=m^{\prime}+\frac{5 \eta m^{2} \gamma_{c}^{2}}{6 a c^{2}},
$$




$$
\begin{gathered}
m_{b}=m_{g} \approx m \gamma_{c}-\frac{3 \eta m^{2} \gamma_{c}}{10 a c^{2}} \approx m+\frac{27 \eta m^{2}}{20 \sqrt{14} a c^{2}}, \quad M \approx m_{b}-\frac{1}{6}\left(7-\frac{27}{2 \sqrt{14}}\right) \frac{\eta m^{2} \gamma_{c}}{a c^{2}} . \\
m^{\prime}<M<m<m_{b}=m_{g} .
\end{gathered}
$$

In (12), the mass-energy of the gravitational and electromagnetic fields outside the matter is equal to $m_{f o}=-\frac{G m_{b}^{2}}{2 a c^{2}}+\frac{q_{b}^{2}}{8 \pi \varepsilon_{0} a c^{2}}=-\frac{5 \eta m^{2} \gamma_{c}^{2}}{6 a c^{2}}$. The difference in masses in (12) and (13) is expressed in units $\frac{\eta m^{2}}{a c^{2}}$ and can also be expressed in terms of $m_{f o}$.

In (13) the mass $m$, which has an auxiliary character, is equal to the product of the mass density $\rho_{0}$ by the body volume, in this case the density $\rho_{0}$ is measured in the reference frames associated with the particles. The gauge mass $m^{\prime}$ refers to the mass-energy of the particles under action of fields, and is associated with the energy gauge and the cosmological constant. The mass $M$ is the invariant mass of the system under Lorentz transformations and it can be considered the inertial mass. The mass $m_{b}$ is greater than other masses because the particles inside the sphere move faster under the influence of the fields and have an increased Lorentz factor [5]. In this case, the mass $m_{b}$ as the total rest mass of particles is equal to the gravitational mass $m_{g}$, since it is the mass $m_{b}$ that is present in the expressions for the strength, scalar potential and energy of the gravitational field outside the sphere [8].

From [12] it follows that

$$
\Delta E=m_{b} c^{2}-M c^{2}
$$

This means that the binding energy $\Delta E$ divided by the square of the speed of light, in view of the relation $m_{b}=m_{g}$, is the difference between the gravitational mass $m_{g}$ and the invariant mass $M$ of the system under consideration.

In (12), taking into account that mass $m^{\prime}$ assumed to be constant, the case is possible when addition of the electric charge to the uniformly charged body starts to decrease the mass $M$. Though transfer of the charge to the body increases the mass $M$ due to the mass $m_{q}$ of the charge carriers, but the electric field energy increases quadratically with respect to 
the charge $q_{b}$, and the contribution of the mass-energy of the field could exceed the contribution from $m_{q}$. For this the following condition must be met:

$$
\frac{q_{b}^{2}}{8 \pi \varepsilon_{0} a c^{2}}>m_{q}
$$

If $q_{b}=N q_{e}, m_{q}=N m_{e}$, where $q_{e}$ and $m_{e}$ denote the charge and mass of one electron, $N$ is the number of electrons transferred to the body, then this condition can be rewritten as the relation for the electric potential, which should take place on the surface of the body:

$$
\frac{q_{b} q_{e}}{8 \pi \varepsilon_{0} a c^{2} m_{e}}>1, \quad \varphi_{a}>\frac{2 c^{2} m_{e}}{q_{e}}=1.02 \times 10^{6} \mathrm{~V}
$$

\section{Conclusion}

In Section 2 we describe the gauge mass $m^{\prime}$, and in Section 3 we consider different masses that characterize the system and express their relation with each other. In (11) we express the relativistic energy in terms of the mass $m_{b}$ and the charge of the system $q_{b}$, and in (7) and (10) - in terms of the mass $m^{\prime}$. The gauge mass $m^{\prime}$ is associated with the energy of particles in the potentials of all the four fields and is expressed in (6).

The difference of $m^{\prime}$ from the invariant inertial mass of the system $M$ in (2) consists in the fact that the mass $M$ according to (12) contains the contribution of the mass-energy of the electromagnetic and gravitational fields in the entire volume outside the system, taken with the opposite sign. The mass-energies of the fields inside the system do not make any contribution, since the acceleration field and the pressure field appear inside the sphere, and in view of (9) the sum of the contributions of all the four fields becomes equal to zero.

In order to describe the mass of the physical system we introduce in (13) five different masses, the lowest of which is $m^{\prime}$ and the largest is the mass $m_{b}$. The mass $m_{b}$ is greater than the invariant mass of the system $M$, because the contribution of the gravitational field into the total energy is negative and exceeds the contributions of other fields for cosmic systems. The mass $m_{b}$ is close in value to the total mass of the matter's baryons and electrons, which is used in the general theory of relativity. However, in our approach the mass $m_{b}$ coincides with the gravitational mass $m_{g}$ and is not equal to the inertial mass $M$, 
in contrast to the general theory of relativity, where $m_{g}=M$. This is due to the fact that we calculated the masses $m_{g}$ and $M$ in the framework of the covariant theory of gravitation.

We can assume that the masses $m_{g}$ and $M$ are likely intended for an external observer, estimating the gravitational and inert properties of the system. The mass $m_{g}$ is associated with the gravitational action exerted by the system on other bodies or with the action exerted by the external bodies on the system under consideration. The invariant mass $M$ of the system is included in the expression for the energy and momentum of the system, and therefore it reflects the inert properties of the system from the point of view of action of nongravitational forces on this system.

The masses $m_{b}$ and $m^{\prime}$ can be significant for the internal observer, who is trying to determine the sum of the invariant masses of the system's particles and the interaction energy of these particles. The mass $m$ allows us to estimate the average density of the particles in their rest reference frame by dividing $m$ by the system's volume.

In addition to masses, the system in question is characterized by two electric charges. One of these charges is given by the expression $q=\rho_{0 q} V$ and is proportional to the volume $V$ of the system and to the invariant charge density $\rho_{0 q}$, measured in the reference frames associated with the moving particles. The other charge $q_{b}$, according to (8), determines the electromagnetic field of the system beyond its limits, and $q_{b}>q$. The difference between these charges is due to the motion of particles and different charge densities of the moving and fixed particles from the standpoint of the theory of relativity.

\section{References}

1. Fedosin S.G. The Concept of the General Force Vector Field. OALib Journal, Vol. 3, pp. 1-15 (2016), e2459. http://dx.doi.org/10.4236/oalib.1102459.

2. Fedosin S.G. Two components of the macroscopic general field. Reports in Advances of Physical Sciences, Vol. 1, No. 2, 1750002, 9 pages (2017). http://dx.doi.org/10.1142/S2424942417500025.

3. Fedosin S.G. Estimation of the physical parameters of planets and stars in the gravitational equilibrium model. Canadian Journal of Physics, Vol. 94, No. 4, pp. 370-379 (2016). http://dx.doi.org/10.1139/cjp-2015-0593. 
4. Fedosin S.G. The Hamiltonian in Covariant Theory of Gravitation. Advances in $\begin{array}{lllllll}\text { Natural Science, } & \text { Vol. 5, } & \text { No. } & 4, & \text { pp. } & 55-75 & \text { (2012). }\end{array}$ http://dx.doi.org/10.3968\%2Fj.ans.1715787020120504.2023.

5. Fedosin S.G. Relativistic Energy and Mass in the Weak Field Limit. Jordan Journal of Physics. Vol. 8, No. 1, pp. 1-16 (2015). http://dx.doi.org/10.5281/zenodo.889210.

6. Fedosin S.G. The virial theorem and the kinetic energy of particles of a macroscopic system in the general field concept. Continuum Mechanics and Thermodynamics, Vol. 29, Issue 2, pp. 361-371 (2016). https://dx.doi.org/10.1007/s00161-016-0536-8.

7. Fedosin S.G. About the cosmological constant, acceleration field, pressure field and energy. Jordan Journal of Physics. Vol. 9, No. 1, pp. 1-30 (2016). http://dx.doi.org/10.5281/zenodo.889304.

8. Fedosin S.G. The Gravitational Field in the Relativistic Uniform Model within the Framework of the Covariant Theory of Gravitation. International Letters of Chemistry, Physics and Astronomy, Vol. 78, pp. 39-50 (2018). http://dx.doi.org/10.18052/www.scipress.com/ILCPA.78.39.

9. Fedosin S.G. The generalized Poynting theorem for the general field and solution of the 4/3 problem. International Frontier Science Letters, Vol. 14, pp. 19-40 (2019). https://doi.org/10.18052/www.scipress.com/IFSL.14.19.

10. Fedosin S.G. The Integral Energy-Momentum 4-Vector and Analysis of 4/3 Problem Based on the Pressure Field and Acceleration Field. American Journal of Modern Physics, Vol. 3, No. 4, pp. 152-167 (2014). https://dx.doi.org/10.11648/j.ajmp.20140304.12.

11. Fedosin S.G. The electromagnetic field in the relativistic uniform model. International Journal of Pure and Applied Sciences, Vol. 4, Issue. 2, pp. 110-116 (2018). http://dx.doi.org/10.29132/ijpas.430614.

12. Fedosin S.G. The binding energy and the total energy of a macroscopic body in the relativistic uniform model. Middle East Journal of Science, Vol. 5, Issue 1, pp. 46-62 (2019). http://dx.doi.org/10.23884/mejs.2019.5.1.06. 